\title{
Is pulmonary artery a dose-limiting organ at risk in non-small cell lung cancer patients treated with definitive radiotherapy?
}

Jie-Tao Ma, Li Sun, Xin Sun, Zhi-Cheng Xiong, Yang Liu, Shu-Ling Zhang, Le-Tian Huang and Cheng-Bo Han*

\begin{abstract}
Purpose: Our previous study suggested that some pulmonary artery (PA) dosimetric parameters were associated with mortality in unresectable non-small cell lung cancer (NSCLC) treated with definitive radiotherapy. The present study aims to analyze the impact of both PA and heart dosimetric parameters on survival of patients with NSCLC treated with definitive conventional fractionated radiotherapy (CFRT) in another independent research center and further determine whether the PA should be considered a dose-limiting organ at risk (OAR) for patients receiving thoracic CFRT.
\end{abstract}

Methods: We performed a retrospective analysis of successive patients with medically inoperable or unresectable NSCLC treated with definitive radiotherapy or chemoradiotherapy from August 2010 to September 2014. Clinical and pathological information, PA and heart dosimetric factors, and follow-up data were collected from each patient's records and evaluated as potential prognostic factors for survival. Survival probabilities were estimated by the KaplanMeier method and compared by the log rank test. Cox proportional hazards regression models were performed to determine the independent predicators of survival. The optimal cutoff points of continuous dosimetric variables were determined by Youden index in receiver operating characteristic (ROC) analysis.

Results: This study analyzed the records of 141 patients, 50.4\% had adenocarcinoma, 71.6\% had stage III disease, and 55\% patients received concurrent chemoradiotherapy. Radiation dose ranged from 60 to 76 Gy in 30-38 fractions. Median follow up was 16.9 months. Median overall survival (OS) was 20.5 months (95\% confidence interval [Cl] 10.3-30.7 months), and 1-, 2-, 3-year OS rates were 75.2\%, 58.2\% and 56\%, respectively. Univariate and multivariate analysis showed that Karnofsky Performance Status (KPS) score, Charlson's Comorbidity Index (CCl), T and N stage, PA invasion grade and the percentage of PA volume that received 40 to 55 Gy (PA V40-55) were significantly associated with OS. No significant associations were found between heart dosimetric factors and OS. Median OS of patients with PA invasion grade 0, 1, 2, and 3 were 41.8, 27.8, 12.7 and 7.5 months, respectively $(P<0.001)$. PA V40, V45, V50 and V55, using thresholds of $80 \%, 68 \%, 45 \%$, and $32 \%$, respectively, were independent predictors for OS.

Conclusions: PA invasion grade and PA V40-55 appear associated with OS in patients with NSCLC treated with definitive CFRT. We propose that PA be considered as a dose-limiting OAR for such patients.

Keywords: Pulmonary artery, Non-small cell lung cancer, Definitive radiotherapy, Organ at risk, Survival

\footnotetext{
* Correspondence: hanchengbo@sj-hospital.org

Department of Oncology, Shengjing Hospital of China Medical University,

Shenyang 110022, China
} 


\section{Background}

Lung cancer is one of the most common cancers worldwide, and non-small cell lung cancer (NSCLC) comprises $85 \%$ of lung cancer cases [1]. Radiotherapy is the primary method of treating patients with medically inoperable or unresectable locally advanced NSCLC (LA-NSCLC) [2-4]; addition of concurrent chemotherapy to radiotherapy (CRT), which is standard care for unresectable LA-NSCLC, achieves a median OS of 17-27 months [5-8]. Nevertheless, some patients suffer early recurrence and severe adverse events, such as radiation-induced pulmonary and esophageal injury, as well as massive hemoptysis.

Optimizing the radiation plan and minimizing radiationinduced toxicities requires limiting the dose to the organs at risk (OAR). Dose-volume parameters of lung, such as V20 and mean lung dose (MLD), are known to play an important role in predicting severe radiation pneumonitis and early deaths [9]. The pulmonary artery (PA) is the most commonly tumor-involved thoracic great vessel $[10,11]$. Patients who had PA invasion by tumor were usually considered to have unresectable disease, though vascular reconstruction has been applied in selected patients. Our previous study of 100 patients with inoperable NSCLC in the United State first categorized the grade of PA invasion by contrast-enhance Computed Tomography (CT) or Positron Emission Tomography (PET) scan and indicated that only 2 out of 4 patients with grade 5 PA invasion experienced massive bleeding caused death, and overall grades of PA invasion were not associated with overall survival in patients with inoperable NSCLC. However, in patients with LA-NSCLC treated with concurrent chemoradiation, the proportion of PA volume that received more than 4560 Gy was associated with shorter OS [12].

The heart is known to be an important OAR for patients receiving thoracic radiotherapy. Postoperative radiation therapy for lung cancer was associated with increased mortality from heart disease [13]. In the RTOG 0617 trial, there were less than 5\% cardiac adverse events ( $\geq$ grade 3 ) in either the high dose or low dose arm, and multivariate analysis showed that the higher proportions of heart volume receiving $\geq$ 5 Gy (H V5) or $\geq 30$ Gy (H V30) were significantly correlated with shorter survival [7]. However, Tucker et al. [14] did not confirm the result of that trial regarding the impact of heart dose volume on OS.

In our previous study [12], the heart was not evaluated in the univariate or multivariate analysis. In addition, data from another independent research center is needed to confirm these findings regarding the PA. Hence, we performed a retrospective analysis to re-evaluate the results of the previous study and verify the impact of dosimetric parameters of the PA and heart on the survival of patients with NSCLC treated with definitive conventional fractionated radiotherapy (CFRT) with or without chemotherapy and to determine whether PA should be considered an OAR and what PA dose/volume limit should recommended for patients receiving definitive CFRT.

\section{Methods \\ Patients}

We retrospectively reviewed the records of successive patients with pathologically confirmed and medically inoperable or unresectable stage I-IIIB NSCLC at Shengjing Hospital between August 2010 and September 2014. Patients who had good Karnofsky Performance Status (KPS) $(\geq 70)$, acceptable forced expiratory volume in $1 \mathrm{~s}$ (FEV1) $(>1.2 \mathrm{~L})$ or FEV1\% (>70\%), and received the definitive conventionally fractionated radiotherapy with or without concurrent chemotherapy were included in this study. Comorbidities, including hypertension, cardiac-cerebral vascular disease, and chronic obstructive pulmonary disease, were calculated by Charlson's Comorbidity Index (CCI) [15, 16]. This study was approved by the local ethics committee of our hospital. All patients provided written informed consent prior to participating in the study.

\section{Radiotherapy parameter extraction}

Radiotherapy was carried out using intensity modulated radiation therapy (IMRT) or 3-dimensional conformal radiatherapy (3DCRT) techniques. Targets contours were defined as follows: gross tumor volume (GTV) included primary lesion (GTVp) and metastatic lymph nodes (GTVnd), which were either pathologically confirmed, had a short diameter $\geq 1 \mathrm{~cm}$, or were PET positive; clinical target volume (CTV) was defined by GTVp plus a 6-8 $\mathrm{mm}$ margin and the involved lymph node region; planning target volume (PTV) was defined by CTV plus a $0.5 \mathrm{~cm}$ margin for axial direction and a 1$1.5 \mathrm{~cm}$ margin for head-foot direction. A total dose of $60-76$ Gy in 30-38 fractions was delivered with 2.0 Gy daily fractions over 6-7.5 weeks using $6 \mathrm{MV}$ photons. The prescribed dose covered $95 \%$ of the PTV. The dose limits for the organs at risk (OAR) were as follows: bilateral lung: V20 (percentage of volume receiving more than $20 \mathrm{~Gy}) \leq 30 \%$ and mean lung dose (MLD) $\leq 20$ Gy; spinal cord: maximum dose of planning organ at risk volume $(\mathrm{PRV}) \leq 48-52$ Gy; heart: V $30 \leq 40-60 \%$ and V $40 \leq 30-50 \%$; esophagus: V50 $\leq 50 \%$. The PA and heart were contoured according to Radiation Therapy Oncology Group (RTOG) 1106 atlas. Dosimetric parameters were extracted from the treatment planning system (Oncentra, Elekta company, Sweden), including mean and maximum doses to the heart or PA as well as the percentage of PA or heart volume that received a specific dose (from V5, 10, 15 to V70 every 5th interval). The evaluation of the grades of PA invasion included primary tumor and metastatic lymph nodes 
invading PA. PA invasion grade criteria were slightly modified from those described previously [12] and are summarized in Table 1. As per these modified criteria, PA invasion was graded as $0,1,2$, and 3 for no, minimal, moderate, and extensive invasion, respectively.

\section{Follow-up}

All patients underwent follow up with chest CT scan every 3 months during the first two years after radiotherapy and every 6 months thereafter. In addition, abdominal CT, bone Emission Computed Tomography and brain Magnetic Resonance Imaging scans were performed every 6 months or as clinically indicated. OS was calculated from the start of radiotherapy until any cause of death or the last date of follow-up. Patients still alive at the last follow-up date (Sep 25, 2015) were censored on that day, and loss to follow-up was considered as a censored event. Progression-free survival (PFS) was calculated from the start of radiotherapy until the first imaging diagnosis of recurrent or progressive disease. Local progression-free survival (LPFS) was based on recurrence within the radiation field.

\section{Statistical analyses}

The potential prognostic factors for univariate analyses of survival included patients characteristics, such as age; gender; score of KPS, CCI; tumor associated factors, such as tumor location, clinical stage, tumor $(\mathrm{T})$ stage, lymph nodal $(\mathrm{N})$ stage, histologic type, and PA invasion grade; treatment associated factors, such as treatment modality (radiotherapy alone or sequential CRT or concurrent CRT), radiotherapy technique (IMRT or 3DCRT), and RT dose; as well as dosimetric parameters, such as minimum dose for 95\% volume of GTV, CTV and PTV (D95), the

Table 1 Grading criteria of pulmonary artery invasion ${ }^{a}$

\begin{tabular}{ll}
\hline Grade & Definition based on CT contrast \\
\hline Grade 0 (no invasion) & No evidence of vessel invasion, \\
& $\geq 1 \mathrm{~mm}$ from the closest pulmonary \\
& vessel wall (presence of a fat plane \\
& between tumor and vessel wall) \\
Grade 1 (minimal invasion) & Tumor invasion with 0 mm to the \\
& closest pulmonary vessel wall, no fat \\
& plane, without presence of narrowing \\
& or truncation of vessels, nor signs of \\
& vessel wall damage (irregularity, \\
& discontinuity or intra-luminal mass \\
& formation) \\
Grade 2 (moderate invasion) & Circumferential involvement with \\
& narrowing or truncation \\
& Tumor invading pulmonary vessel \\
Grade 3 (extensive invasion) & extensively with any sign of vessel \\
& wall damage: irregularity, discontinuity \\
& or intra-luminal mass formation or \\
massive haemorrhage due to the & tumor invading pulmonary artery \\
&
\end{tabular}

${ }^{\mathrm{a}}$ Revised from [12] percentage of PA volume (PA V5, 10, 15, and 20-70) and heart volume ( $\mathrm{H} \mathrm{V5}, 10$, and 15-70) that received a specific radiation dose (Table 2). Pearson correlation coefficient was used to examine the correlation between potential prognostic factors. Survival was estimated using the Kaplan-Meier method and curves were compared using the log rank test. Both univariate and multivariate analyses were performed to evaluate associations between potential prognostic factors and OS. Multivariable Cox proportional hazard models were used to calculate adjusted hazard ratios (HRs) and their 95\% confidence intervals (CIs); variables likely to be associated with OS, based on $P$ values of less than 0.1 from the univariate analysis, were included in these models. Simultaneous inclusion in the same multiple variable regression model of two highly correlated variables (correlation co-efficient greater than 0.7 ) was considered inappropriate. Consequently, for multivariable analysis, such variables were entered separately, one by one, with other independent variables. The optimal cutoff points of significant continuous variables were determined by Youden index in receiver operating characteristic (ROC) analysis. Area under the curve (AUC) determined by ROC analysis was used to estimate the predictive ability of covariates for survival status; cutoff values were evaluated and confirmed by repeated Kaplan-Meier and multivariate Cox analyses. The software of IBM SPSS version 21.0 was used for statistical analysis, a $P$ value less than 0.05 was considered statistically significant.

\section{Results}

\section{Characteristics of patients}

One hundred forty-one patients with pathologically confirmed stage I-IIIB NSCLC were eligible for this study. Patient characteristics are summarized in Table 2.

\section{Correlation of potential prognostic factors}

The correlations among clinicopathologic factors and dosimetric parameters were calculated. RT dose was not correlated with GTV, CTV and PTV; Pearson correlation coefficients were $0.171,0.121$ and 0.064 , respectively, all $P$ values were $>0.05$. Both $\mathrm{T}$ stage and $\mathrm{N}$ stage were not correlated with volumes of GTV, CTV or PTV or D95 to target volumes, Pearson correlation coefficients ranged from -0.126 to -0.291 , all $P$ values were $>0.05$. In addition, no correlations were noted between heart dosimetric parameters and target volumes $(P>0.05)$. Only weak correlations were found between target volumes and some PA dosimetric parameters (PA V35-60) and between $\mathrm{H}$ V40-65 and PA V35-65, but all Pearson correlation coefficients were less than $0.4, P<0.05$.

\section{Results of univariate survival analysis}

The median follow-up for the entire cohort was 16.9 months (range: 6.5 to 42.0 months). Median PFS, LPFS, and OS 
Table 2 Clinicopathologic characteristic

\begin{tabular}{|c|c|c|c|}
\hline Characteristic & Number (\%) & Characteristic & Number (\%) \\
\hline Age (years) & & RT modality & \\
\hline Median (range) & $60(39-85)$ & RT alone & 55 (39\%) \\
\hline$<60$ & $72(5.1)$ & concurrent CRT & 47 (33.3\%) \\
\hline$\geq 60$ & $69(48.9)$ & sequential CRT & 39 (27.7\%) \\
\hline Gender & & Tumor stage & \\
\hline Male & 97 (68.8\%) & $\mathrm{T} 1$ & $26(18.4 \%)$ \\
\hline Female & $44(31.2 \%)$ & $\mathrm{T} 2$ & $46(32.6 \%)$ \\
\hline KPS (70-100) & & T3 & $38(27 \%)$ \\
\hline $70-80$ & $5(3.5 \%)$ & T4 & $31(22 \%)$ \\
\hline $80-90$ & 28 (19.9\%) & Lymph nodes stage & \\
\hline $90-100$ & $108(76.6)$ & No & $42(29.8 \%)$ \\
\hline Smoking & & $\mathrm{N} 1$ & $48(34 \%)$ \\
\hline No & 68 (48.2\%) & N2 & 32 (22.7\%) \\
\hline Yes & $73(51.8 \%)$ & N3 & $19(13.5 \%)$ \\
\hline Weight loss (\%) & & Clinical stage & \\
\hline Median (range) & $3(0-10)$ & 1 & $5(3.5 \%)$ \\
\hline$\leq 3 \%$ & $72(51.1)$ & $\|$ & $47(33.3 \%)$ \\
\hline$>3 \%-10 \%$ & $69(48.9)$ & $\| \mathrm{A}$ & $62(44 \%)$ \\
\hline COPD & & $\| 11 B$ & $27(19.1 \%)$ \\
\hline No & $130(92.2 \%)$ & PA invasion grade & \\
\hline Yes & $11(7.8 \%)$ & Grade 0 & $49(34.8 \%)$ \\
\hline CVD & & Grade 1 & $36(25.5 \%)$ \\
\hline No & $108(76.6 \%)$ & Grade 2 & $37(26.2 \%)$ \\
\hline Yes & $33(23.4 \%)$ & Grade 3 & 19(13.5\%) \\
\hline Hypertension & & Target volume (cc) & \\
\hline No & 109 (77.3\%) & & \\
\hline Yes & $32(22.7 \%)$ & GTV & $43.6(9-400.9)$ \\
\hline $\mathrm{CCl}$ & & CTV & $92.2(12.56-843.6)$ \\
\hline Median (range) & $3(0-7)$ & PTV & $213.7(23.5-1025.5)$ \\
\hline$<3$ & $62(44.0 \%)$ & \multirow{2}{*}{$\begin{array}{l}\text { D95 to targets (Gy) } \\
\text { median (range) }\end{array}$} & \\
\hline$\geq 3$ & 79 (56.0\%) & & \\
\hline Tumor location & & D95 to GTV & $64.9(48.1-74.0)$ \\
\hline Central & $116(82.3 \%)$ & D95 to CTV & $63.1(46.4-71.9)$ \\
\hline Peripheral & $25(17.7 \%)$ & D95 to PTV & $60.1(45.6-69.9)$ \\
\hline Pathology & & $D_{\max }$ to $P A(G y)$ & \\
\hline Adenocarcinoma & 71 (50.3\%) & Median (range) & $65.8(0.6-76.2)$ \\
\hline Squamous & $61(43.3 \%)$ & $D_{\text {mean }}$ to $P A$ (Gy) & \\
\hline Large-cell \& NOS & $9(6.4 \%)$ & Median (range) & $37.9(0-71.3)$ \\
\hline RT technique & & $D_{\max }$ to Heart (Gy) & \\
\hline IMRT & $52(36.9 \%)$ & Median (range) & $56(0.2-65.7)$ \\
\hline 3DCRT & 89 (63.1\%) & $D_{\text {mean }}$ to Heart (Gy) & \\
\hline RT dose (Gy) & & Median (range) & $5.2(0-34.9)$ \\
\hline Median (range) & $66(60-76)$ & & \\
\hline$<66$ & $62(44.0 \%)$ & & \\
\hline$\geq 66$ & $79(56.0 \%)$ & & \\
\hline
\end{tabular}

KPS Karnofsky performance status, COPD chronic obstructive pulmonary disease, CVD Cardiac vascular disease, CCI Charlson comorbidity index, NOS NSCLC not otherwise specified, IMRT intensity modulated radiation therapy, 3DCRT three-dimensional conformal radiation therapy, RT radiotherapy, Chemo Chemotherapy, GTV gross tumor volume, CTV clinical target volume, PTV planning target volume, D95 minimum dose to $95 \%$ volume of targets, $P A$ pulmonary artery, $c C$ cubic centimeter, Gy gray, Dmax Maximum dose, Dmean Mean dose 
were 8.4 months, 12.4 months, and 20.5 months (95\% CI: 9.58-31.42 months), respectively, and the 1-, 2- and 3-year OS rates were $75.9 \%, 58.2 \%$ and $56 \%$, respectively (Fig. 1). Univariate analysis showed that age, gender, status of smoking, weight loss, tumor location, treatment modality, RT dose, D95 to target volumes, maximum and mean dose to the PA or heart were not related with OS. However, KPS score, $\mathrm{T}$ stage, $\mathrm{N}$ stage, clinical stage, CCI, PA invasion grade, GTV, CTV, PTV, PA V20, 25-65 and H V30, 35-45 were significantly associated with median OS $(P<0.1)$ (Table 3$)$.

\section{Results of multivariate survival analysis}

Multivariate analysis indicated that the clinicopathologic factors significantly associated with shorter OS were lower KPS score $(<85)$, higher CCI $(\geq 3)$, higher $\mathrm{T}$ (T3, T4) stage, $\mathrm{N}(\mathrm{N} 2, \mathrm{~N} 3)$ stage, and higher grade of PA invasion. Median OS was 13.1 months vs. 32.7 months in patients with $\mathrm{KPS}<85$ vs. $\geq 85$, respectively; was 10.9 months vs. 37.9 months in $\mathrm{CCI} \geq 3$ vs. < 3, respectively; was 13.5 months vs. 41.8 months in T3, 4 stage vs. T1, 2 stage, respectively; and was 19.3 months vs. 27.8 months in N2, 3 stage vs. N0, 1 stage, respectively. All $P$ values were less than 0.05 . In addition, the median OS and 2-year survival rates were 41.8 months and 79.6\%, 27.8 months and 66.67\%, 12.7 months and $37.84 \%$, and 7.5 months and zero, respectively, for no invasion (grade 0), minimal (grade 1), moderate (grade 2), and extensive invasion (grade 3), $P<0.05$ (Fig. 2 and table 4). Two patients with grade 3 PA invasion (2/19, $10.5 \%$ ) died of massive bleeding after radiotherapy, and no other bleeding events or rupture of great vessel were observed during or after radiotherapy.

With regard to dosimetric factors, multivariate analysis demonstrated that volumes of targets including GTV, CTV, PTV and the percentages of heart volume that received a specific radiation dose, were not significantly associated with OS. However, PA V40-55 Gy were significantly associated with OS independent of KPS and stage. According to the ROC curves, the best cutoff values for PA V40, 45, 50 and 55 predictive of OS were $80 \%, 68 \%, 45 \%$, and $32 \%$, respectively. Median OS rates were 14 months vs. 27.8 months, respectively in patients with PA V $40 \geq 86 \%$ vs. $<86 \%, P<0.0001$; were 13.5 months vs. 37.9 months, respectively in patients with PA V45 $\geq 68 \%$ vs. $<68 \%, P<0.0001$; were 14.2 months vs. 32.7 months, respectively in patients with PA V50 $\geq 45 \%$ vs. $<45 \%, P<0.0001$; and were 10.9 months vs. 41.8 months, respectively in patients with PA V55 $\geq 32 \%$ vs. $<32 \%, P<0.0001$ (Fig. 3a-d). The dose volume histograms (DVH) and dose-curves of four patients presenting with high grades of PA invasion and PA V40-55 are shown in Fig. 4a-d.

\section{Discussion}

The present study demonstrated that the score of KPS, CCI, stages of $\mathrm{T}$ and $\mathrm{N}, \mathrm{PA}$ invasion grade, as well as percentage of PA volumes that received 40-55 Gy were independent prognostic factors of OS for patients with NSCLC treated with definitive CFRT. PA invasion grade was significantly associated with OS, though it was not associated with bleeding during or after radiotherapy. In

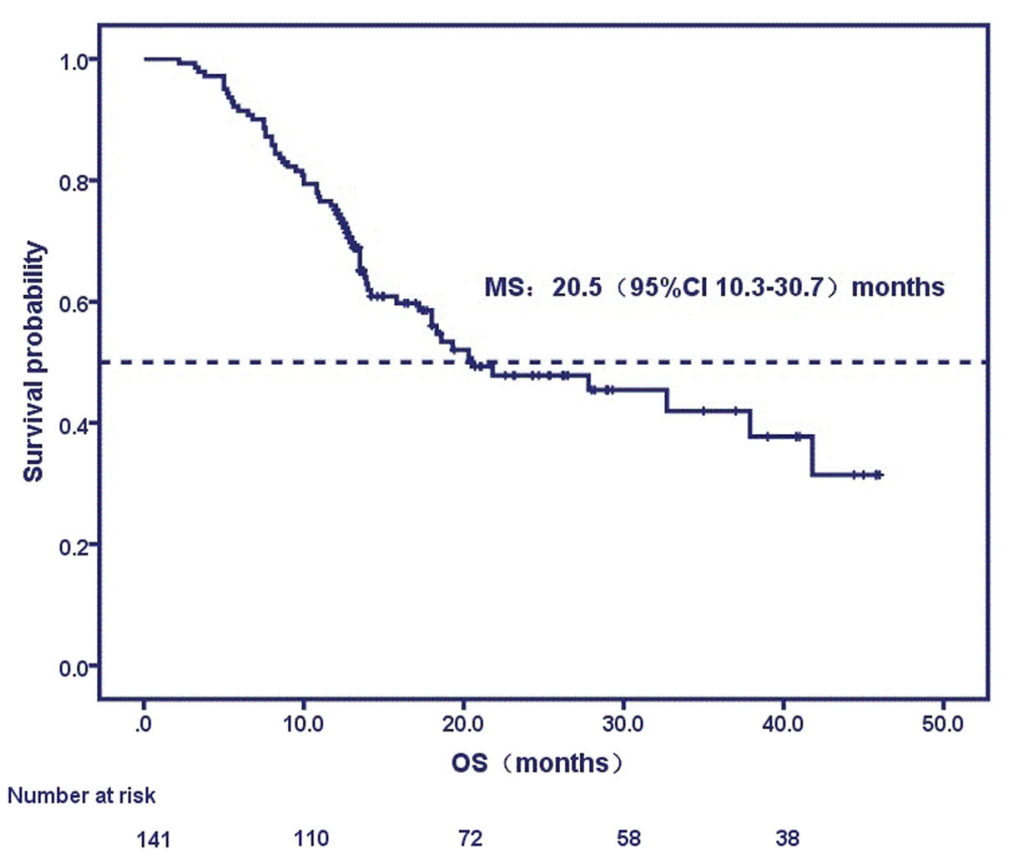

Fig. 1 Curve of overall survival (OS) 
Table 3 The result of multivariate analysis of significative potential prognostic factors associated with overall survival by using univariate analysis

\begin{tabular}{|c|c|c|c|c|c|}
\hline & \multicolumn{2}{|l|}{ Univariate } & \multicolumn{3}{|c|}{ Multivariate } \\
\hline & Variables & $P$ value & $P$ value & $\mathrm{HR}$ & $95 \% \mathrm{Cl}$ \\
\hline \multirow[t]{5}{*}{ Categorical Variables } & $\mathrm{CCl}$ & 0.00001 & 0.00001 & 1.816 & $1.386-2.378$ \\
\hline & T-stage & 0.0004 & 0.0001 & 3.160 & $1.944-5.138$ \\
\hline & N-stage & 0.007 & 0.001 & 1.907 & $1.315-2.767$ \\
\hline & Clinical stage & 0.001 & 0.493 & 0.697 & $0.248-1.96$ \\
\hline & PA invasion grade & 0.028 & 0.0001 & 2.172 & $1.467-3.216$ \\
\hline \multirow[t]{20}{*}{ Continuous Variables } & KPS & 0.001 & 0.043 & 0.934 & $0.886-0.936$ \\
\hline & GTV & 0.015 & 0.066 & 0.986 & $0.975-0.997$ \\
\hline & CTV & 0.045 & 0.07 & 1.01 & $1.003-1.017$ \\
\hline & PTV & 0.081 & 0.203 & 0.998 & $0.995-1.001$ \\
\hline & PA V10 & 0.099 & 0.698 & 0.275 & $0-16.913$ \\
\hline & PA V15 & 0.098 & 0.792 & 0.309 & $0-15.602$ \\
\hline & PA V20 & 0.084 & 0.678 & 1.691 & $0-4.357$ \\
\hline & PA V25 & 0.094 & 0.468 & 0.022 & $0-6.387$ \\
\hline & PA V30 & 0.002 & 0.553 & 8.474 & $0.007-9.855$ \\
\hline & PA V35 & 0.001 & 0.111 & 2.367 & $0.271-2.967$ \\
\hline & PA V40 & 0.001 & 0.043 & 2.113 & $1.014-4.936$ \\
\hline & PA V45 & 0.0004 & 0.0001 & 2.660 & $1.089-5.717$ \\
\hline & PA V50 & 0.001 & 0.0001 & 1.203 & $0.062-2.056$ \\
\hline & PA V55 & 0.002 & 0.05 & 1.489 & $0.098-2.096$ \\
\hline & PA V60 & 0.005 & 0.191 & 0.076 & $0.002-3.621$ \\
\hline & PA V65 & 0.027 & 0.149 & 0.069 & $0.002-2.598$ \\
\hline & H V30 & 0.062 & 0.148 & 0 & $0-4.075$ \\
\hline & H V35 & 0.059 & 0.227 & 1.725 & $0-9.681$ \\
\hline & H V40 & 0.035 & 0.755 & 0.001 & $0-5.625$ \\
\hline & H V45 & 0.092 & 0.923 & 5.765 & $0-12.916$ \\
\hline
\end{tabular}

CCI Charlson comorbidity index, KPS karnofsky performance status, GTV gross tumor volume, CTV clinical target volume, $P T V$ planning target volume, D95 minimum dose to $95 \%$ volume of targets, $P A$ pulmonary artery, PA V5, 10-70: the percentage of PA volume receiving 5,10 to $70 G y, P A_{\text {max }}$ the maximum dose to $\mathrm{PA}, P A_{\text {mean }}$ the mean dose to PA, H V5,10-75: the percentage of heart volume receiving 5,10 to $70 \mathrm{~Gy}, H_{\max }$ the maximum dose to heart, $H_{\text {mean }}$ the mean dose to heart

addition, patients with PA V40 > 80\%, V45 > 68\%, V50 > $45 \%$ or V $55>32 \%$ had a significantly shorter OS. The results further confirmed our previous conclusions [12] and suggested that the PA should be regarded as an OAR during conventionally fractionated thoracic radiotherapy or chemoradiotherapy, and that the grade of PA invasion and PA dosimetric parameters V40-55 could be used to predict survival for those patients with NSCLC who received definitive CFRT.

Radiation induced lung injury, esophagitis, and heart injuries are the most frequent radiation related toxicities and have been widely investigated recently. The RTOG 0617 trial [7] revealed that high dose radiotherapy given to patients with LA-NSCLC failed to improve local control and prolong OS compared with standard dose radiotherapy. The possible damage to the local immune microenvironment of the field receiving high dose radiation, and the radiation related toxicities might be the major reason why patients with high dose radiotherapy had shorter survival. In this trial, $\mathrm{H}$ V5 and $\mathrm{H}$ V30, but not radiation modalities were correlated with OS [7]; The present study also evaluated the relationship between OS and heart dosimetric parameters including maximum or mean dose to the heart, or the percentage of heart volume that received a specific dose (H V5-70). However, no significant associations were found between them, although the radiation related heart injury was not evaluated herein. The reason for this finding might be attributed to relatively strict radiation dose constraints for heart that were used in our study. Our results were in accordance with Tucker et al's study [14], which found that heart dosimetric parameters did not significantly affect survival. 


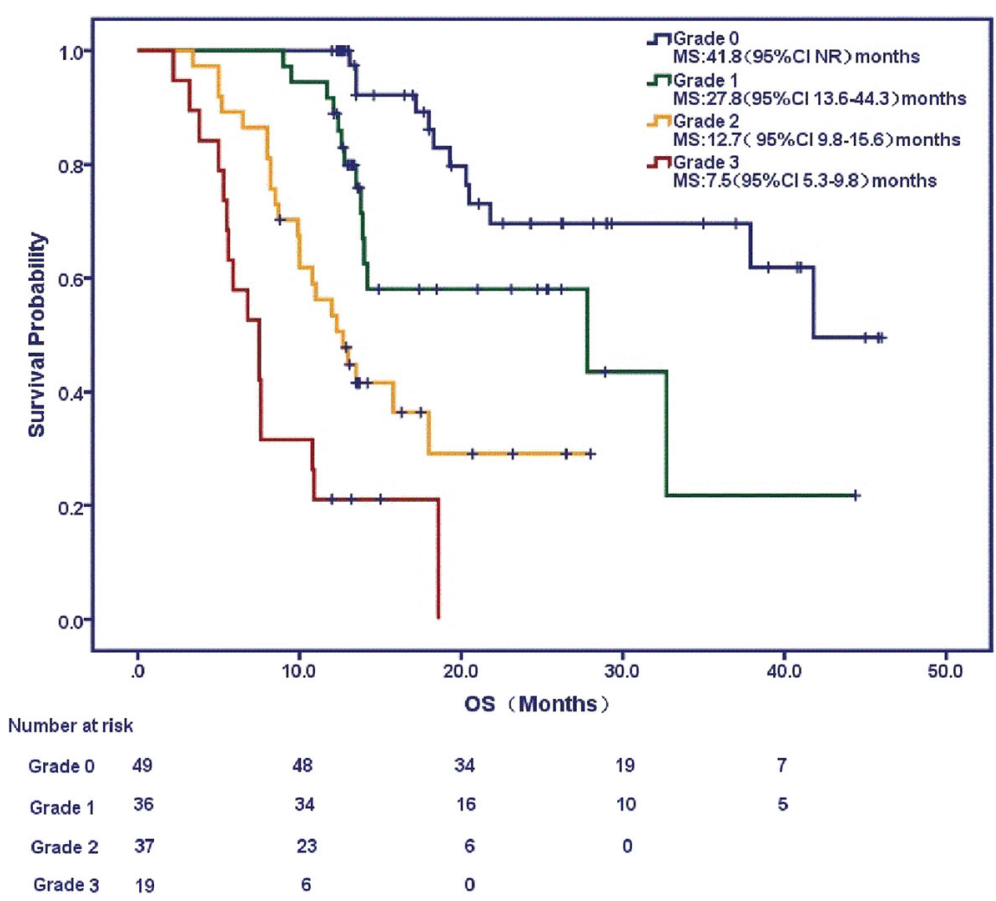

Fig. 2 Curve of overall survival (OS) by pulmonary artery (PA) invasion grade: grade 1 (no invasion), grade 2 (minimal invasion), grade 3 (moderate invasion), and grade 4 (extensive invasion)

A great vessel is deemed as a dose-limiting organ when lesions are given hypofractionated or stereotactic body radiotherapy (SBRT) [17]. Some researchers thought there were greater risks of severe hemoptysis and early mortality among patients who had severe PA invasion during or after radiotherapy. A previous study stated that vessel rupture may result from the penetration of the tumor into the vessel wall itself [18]. However, a later study [19] showed that this explanation might be implausible because penetration of a tumor into the wall of an elastic artery is so unusual except in the following circumstances: the vessel itself (e.g., atherosclerosis), had exposed vasa vasorum during surgery and damage to the adventitia caused by ulcer, fistulas or infection around artery that were independent of radiotherapy [19]. Another study showed that toxicity to the aorta after reirradiation was relatively rare, even when maximum composite doses to the aorta exceeded 100 Gy [20].
Recently, our study [12] first proposed grading criteria for PA invasion from grade 0 to grade 5 , describing the distance of tumor to the PA, degree of circumferential involvement of the PA, and status of vessel wall damage. In that study, we found patients with PA invasion of grade $0-1$ had a longer median OS than those with grade $2-5$ (33.4 months vs. 18.3 months, $P=0.242$ ), and two of the 4 patients with grade 5 PA invasion died suddenly from massive hemorrhage at 3 and 4.5 months after completion of radiotherapy. To facilitate its clinical use, we simplified the grading system to grade $0,1,2,3$ for no, minimal, moderate, and extensive invasion, respectively. In this group of patients, we found a significant relationship between grade of PA invasion and survival. Two patients with extensive PA invasion died of massive bleeding after radiotherapy. We believe that a higher grade of PA invasion indicates worse clinicopathologic behavior and prognosis, though few bleeding events were observed during or after radiotherapy in both studies.

Table 4 Overall survival (OS) of different grade of PA invasion

\begin{tabular}{lllll}
\hline Grade & $\mathrm{n}$ & Median OS (95\%Cl) (months) & 1-year OS rate (\%) & 2-year OS rate (\%) \\
\hline 0 & 49 & $41.8(-)$ & 100 & 79.6 \\
1 & 36 & $27.8(19.96-32.36)$ & 91.67 & 66.67 \\
2 & 37 & $12.7(9.85-15.56)$ & 54.05 & 37.84 \\
3 & 19 & $7.5(5.25-9.75)$ & 21.05 & 0 \\
\hline
\end{tabular}

*P $=0.0003$ for trend 


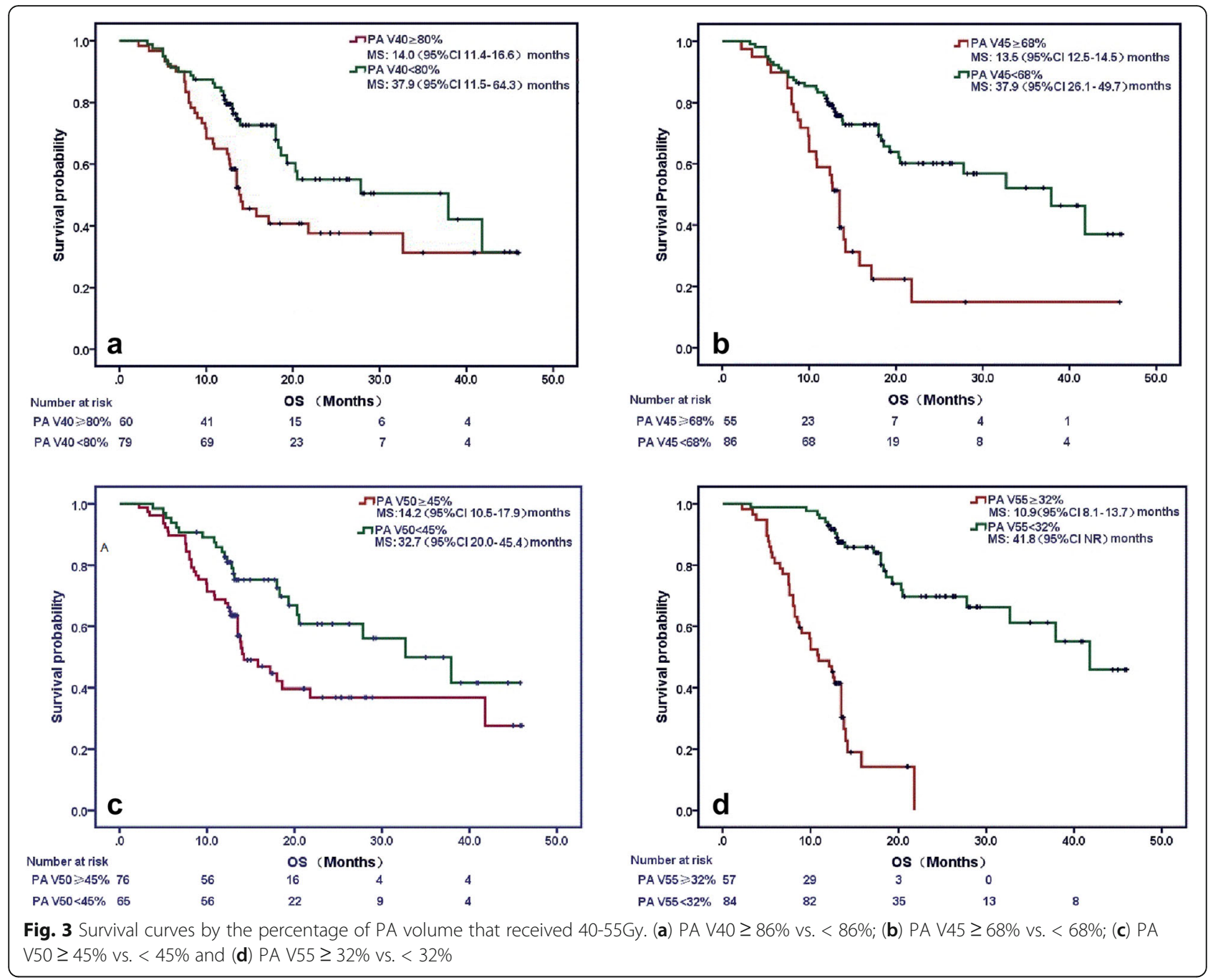

In the future, reliable imaging or pathological assessment of great vessel rupture needs to be developed to confirm this assumption.

The present study also found that the percentages of PA volume that received 40-55 Gy were significantly associated with OS regardless of target volume, KPS, CCI and stage. Especially, patients with PA V45 > 68\%, and those with PA V $55>32 \%$ had a significantly shorter OS. Interestingly, the results were highly consistent with our previous study in which high dose volume of PA V45-60 was predictive of shorter OS, and the cut-off values of PA V45 and V60 were $70 \%$ and $37 \%$, respectively. Both studies suggest that PA V45 (68-70\%) is a better cut-off value predictive of shorter OS in patients with LA-NSCLC treated with CFRT. Our findings differed from those using SBRT, in which maximum dose exceeding 50 Gy to the PA was related with massive hemoptysis and caused death [21]; in our study, when CFRT was administrated, the maximum dose to the PA was not related with OS.
In the present study, we found that GTV, CTV, PTV and dose coverage of target volumes were not associated with OS, which seemed contradictory to the effect of the factors $\mathrm{T}$ or $\mathrm{N}$ stage on OS, because a higher $\mathrm{T}$ or $\mathrm{N}$ stage often results in a larger target volume. However, we did not see any correlation between $\mathrm{T}$ or $\mathrm{N}$ stage and the target volumes GTV, CTV or PTV. Correlation analysis revealed only weak correlations between target volumes and PA V40-V55, thus ruling out an influence of target volume on PA volume dose. Certainly, our study may be too small to distinguish the independent or synthetic effect of heart and PA dosimetric parameters on survival. Future prospective studies with larger numbers of patients are needed to pursue the combined effects of heart and PA dosimetry. In addition, in our study only two patients with extensive PA invasion died of fatal hemoptysis after radiotherapy during follow-up. Hence, it appears that in this cohort, these rare outcomes, such as fatal hemoptysis or massive bleeding, are not good end 

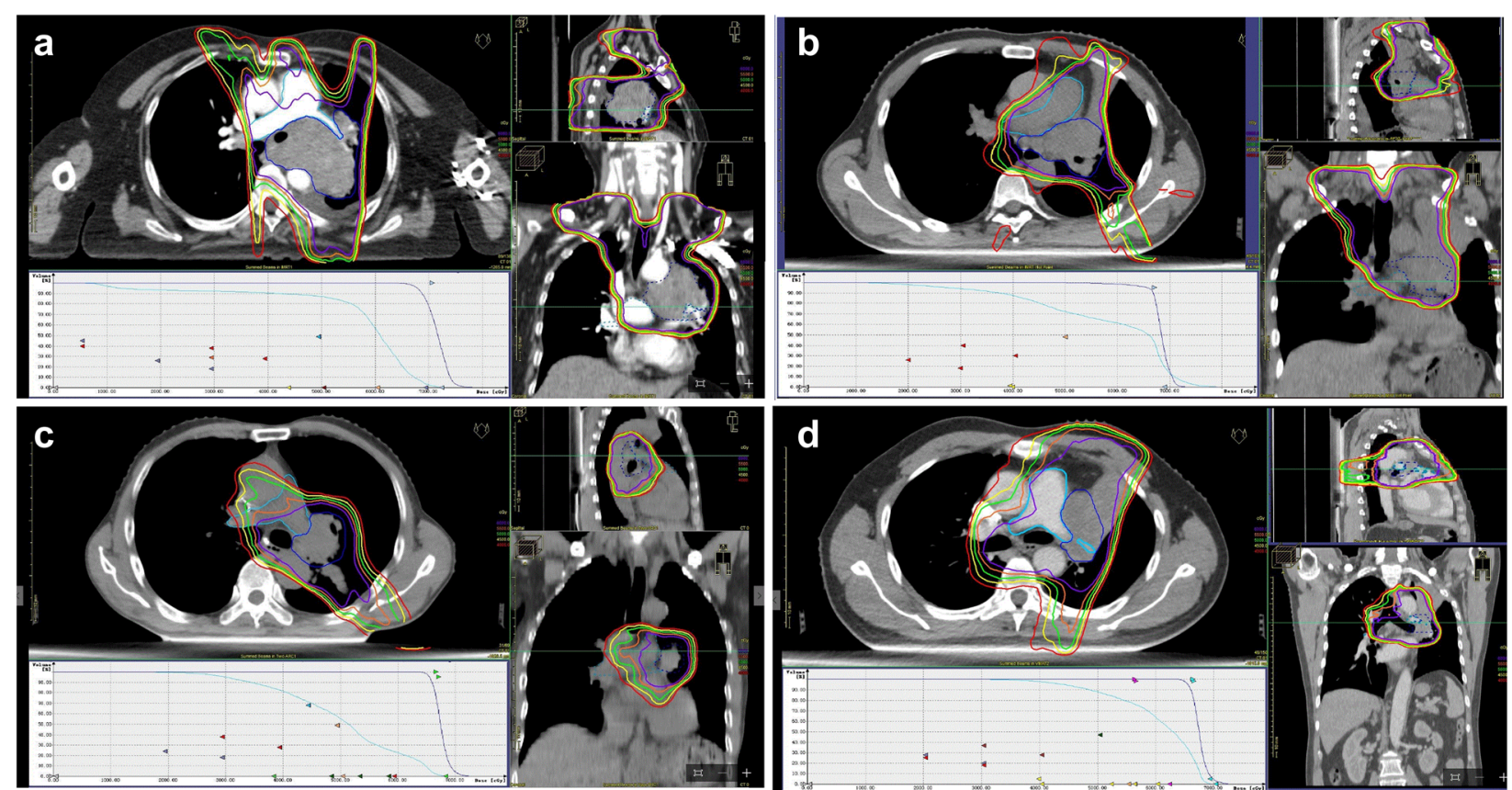

Fig. 4 Four patients with high grade of pulmonary artery (PA) invasion and high PA V40-55 values had short overall survival (OS). Dose volume histograms (DVH) of PA (azure) and PTV (blue), as well as isodose curves of 40 Gy (red), 45 Gy (yellow), 50 Gy (green), 55 Gy (orange), and 60 Gy (purple) in axial, sagittal, and coronal views are shown. PA V40-60 was the percentage of PA volume of PA that received 40-60 Gy. a A patient who was diagnosed with non-small cell lung cancer (NSCLC) (T4N3M0 and PA invasion grade 3) died of massive bleeding approximately 6 months after radiotherapy. PA V40, V45, V50, V55, and V60 were 90\%, 89.02\%, 86.89\%, 81.03\%, and 56.81\%, respectively. b A patient who was diagnosed with NSCLC (T2N2M0 and PA invasion grade 1) survived 9.9 months after radiotherapy. PA V40, V45, V50, V55, and V60 were 87.46\%, 80.09\%, 72.36\%, 66.31\%, and 61.19\%, respectively. c A patient who was diagnosed with NSCLC (T3N0M0 and PA invasion grade 2) survived 8.7 months after radiotherapy. PA V40, V45, V50, V55, and V60 were $81.28 \%, 69.78 \%, 55.03 \%, 33.2 \%$, and 22.72\%, respectively. d A Patient who was diagnosed with NSCLC (T4N1M0 and PA invasion grade 3) survived 5.5 months after radiotherapy. PA V40, V45, V50, V55, and V60 were 97.81\%, 94.12\%, $87.83 \%, 79.45 \%$, and $64.06 \%$, respectively

points guide recommendations for dosimetric constraints for this OAR. PA grading variation from the start of radiotherapy to death may be an alternative end point. However, this is not evaluated in the present study because the accurate PA grading variation largely depends on reliable imaging or pathological analysis of abnormalities, such as ulcer, fistula, and stricture. On the other hand, we believe that the PA is an OAR similar to the heart, and that higher dose-volume irradiation to the PA might also cause serious consequences such as pericarditis, arrhythmia, and fatal hemoptysis. Future studies are needed to validate these postulations.

\section{Conclusion}

PA invasion grade and PA V40-55 were independent factors predictive of OS regardless of KPS, CCI and stage in patients with NSCLC treated with definitive CFRT. We proposed that the PA should be regarded as an organ at risk relevant to target delineation and dose limitation during CFRT. Limiting moderate-to-high PA dose volume, especially PA V45, is recommended due to its better ability to predict survival.

\section{Abbreviations}

3DCRT: 3-Dimensional conformal radiotherapy; AUC: Area under the curve; CCI: Charlson's comorbidity index; CFRT: Conventional fractionated radiotherapy; COPD: Chronic obstructive pulmonary disease; CRT: Concurrent chemotherapy to radiotherapy; CT: Computed tomography; CTV: Clinical target volume; CVD: Cardiac vascular disease; FEV1: Forced expiratory volume in $1 \mathrm{~s}$; GTV: Gross tumor volume; IMRT: Intensity modulated radiation therapy; KPS: Karnofsky performance status; LA-NSCLC: Locally advanced nonsmall cell lung cancer; LPFS: Local progression-free survival; MLD: Mean lung dose; NSCLC: Non-small cell lung cancer; OAR: Organ at risk; OS: Overall survival; PA: Pulmonary artery; PET: Positron emission tomography; PFS: Progression-free survival; PRV: Planning organ at risk volume; PTV: Planning target volume; ROC: Receiver operating characteristic; RTOG: Radiation therapy oncology group; SBRT: Stereotactic body radiotherapy

\section{Acknowledgements}

Not applicable.

\section{Funding}

This study was supported by a grant from the National Natural Science Foundation of China (grant No. 81501990).

\section{Availability of data and materials}

The authors declare that all the data supporting the findings of this study are available within the article.

Authors' contributions

$\mathrm{CH}$ and JM designed the project. LS, XS, SZ and LH contributed to acquisition of data and data analysis. $Z X$ and $Y L$ contributed to methodology 
of the process. JM and LS developed the software tools to analyze the data. $\mathrm{CH}$ was the Senior Author who oversaw the project. All authors read and approved the final manuscript.

\section{Competing interests}

The authors declare that they have no competing interests.

\section{Consent for publication}

Not applicable.

\section{Ethics approval and consent to participate}

Not applicable.

Received: 18 October 2016 Accepted: 24 January 2017

Published online: 01 February 2017

\section{References}

1. Goeckenjan G, Sitter H, Thomas M, Branscheid D, Flentje M, Griesinger F, et al. German Cancer Society. Prevention, diagnosis, therapy, and follow-up of lung cancer: interdisciplinary guideline of the German Respiratory Society and the German Cancer Society. Pneumologie. 2011;65(1):39-59. doi:10.1055/s-0030-1255961.

2. Zhao L, West BT, Hayman JA, Lyons S, Cease K, Kong FM. High radiation dose may reduce the negative effect of large gross tumor volume in patients with medically inoperable early-stage non-small cell lung cancer. Int J Radiat Oncol Biol Phys. 2007;68(1):103-10.

3. Pijls-Johannesma M, Houben R, Boersma L, Grutters J, Seghers K, Lambin P, et al. High-dose radiotherapy or concurrent chemo-radiation in lung cancer patients only induces a temporary, reversible decline in QoL. Radiother Oncol. 2009;91(3):443-8. doi:10.1016/j.radonc.2009.02.010.

4. Hayakawa K, Mitsuhashi N, Katano S, Saito Y, Nakayama Y, Sakurai H, et al. High-dose radiation therapy for elderly patients with inoperable or unresectable non-small cell lung cancer. Lung Cancer. 2001;32(1):81-8.

5. Curran WJ Jr, Paulus R, Langer CJ, Komaki R, Lee JS, Hauser S, et al. Sequential vs. concurrent chemoradiation for stage III non-small cell lung cancer: randomized phase III trial RTOG 9410. J Natl Cancer Inst. 2011;103(19):1452-60. doi:10.1093/jnci/djr325. Erratum in: J Natl Cancer Inst. 2012;104(1):79.

6. Aupérin A, Le Péchoux C, Rolland E, Curran WJ, Furuse K, Fournel P, et al. Meta-analysis of concomitant versus sequential radiochemotherapy in locally advanced non-small-cell lung cancer. J Clin Oncol. 2010;28(13):2181-90. doi:10.1200/JCO.2009.26.2543.

7. Bradley JD, Paulus R, Komaki R, Masters G, Blumenschein G, Schild S, et al. Standard-dose versus high-dose conformal radiotherapy with concurrent and consolidation carboplatin plus paclitaxel with or without cetuximab for patients with stage IIIA or IIIB non-small-cell lung cancer (RTOG 0617): a randomised, two-by-two factorial phase 3 study. Lancet Oncol. 2015; 16(2):187-99. doi:10.1016/S1470-2045(14)71207-0.

8. Senan S, Brade A, Wang LH, Vansteenkiste J, Dakhil S, Biesma B, et al. PROCLAIM: randomized phase III trial of Pemetrexed-cisplatin or etoposidecisplatin plus thoracic radiation therapy followed by consolidation chemotherapy in locally advanced nonsquamous Non-small-cell lung cancer. J Clin Oncol. 2016:34(9):953-62. doi:10.1200/JCO.2015.64.8824.

9. Marks LB, Bentzen SM, Deasy JO, Kong FM, Bradley JD, Vogelius IS, et al. Radiation dose-volume effects in the lung. Int J Radiat Oncol Biol Phys. 2010;76(3 Suppl):S70-6. doi:10.1016/j.jijobp.2009.06.091.

10. Yamashita M, Komori E, Sawada S, Suehisa H, Nozaki I, Kurita A, et al. Pulmonary angioplastic procedure for lung cancer surgery. Gen Thorac Cardiovasc Surg. 2010;58(1):19-24. doi:10.1007/s11748-009-0462-9.

11. Nagayasu T. Importance of follow-up inspection after pulmonary angioplastic procedures for lung cancer surgery. Gen Thorac Cardiovasc Surg. 2010;58(1):1-2. doi:10.1007/s11748-009-0508-z.

12. Han CB, Wang WL, Quint L, Xue JX, Matuszak M, Ten Haken R, et al. Pulmonary artery invasion, high-dose radiation, and overall survival in patients with non-small cell lung cancer. Int J Radiat Oncol Biol Phys. 2014;89(2):313-21. doi:10.1016/j.ijrobp.2014.02.011.

13. Lally BE, Detterbeck FC, Geiger AM, Thomas Jr CR, Machtay M, Miller $A A$, et al. The risk of death from heart disease in patients with nonsmall cell lung cancer who receive postoperative radiotherapy: analysis of the Surveillance, Epidemiology, and End Results database. Cancer. 2007;110(4):911-7.
14. Tucker SL, Liu A, Gomez D, Tang LL, Allen P, Yang J, et al. Impact of heart and lung dose on early survival in patients with non-small cell lung cancer treated with chemoradiation. Radiother Oncol. 2016;119(3):495-500. doi:10.1016/j.radonc.2016.04.025

15. Charlson ME, Pompei $P$, Ales $K L$, Mackenzie CR. A new method of classifying prognostic comorbidity in longitudinal studies: development and validation. J Chronic Dis. 1987;40(5):373-83.

16. Charlson M, Szatrowski TP, Peterson J, Gold J. Validation of a combined comorbidity index. J Clin Epidemiol. 1994;47(11):1245-51.

17. Xue J, Kubicek G, Patel A, Goldsmith B, Asbell SO, LaCouture TA. Validity of current stereotactic body radiation therapy dose constraints for aorta and major vessels. Semin Radiat Oncol. 2016;26(2):135-9. doi:10.1016/j.semradonc. 2015.11.001.

18. Dibbell DG, Gowen GF, Shedd DP. Observations on postoperative carotid hemorrhage. Am J Surg. 1965:109:765-70.

19. Fajardo LF, Lee A. Rupture of major vessels after radiation. Cancer. 1975;36(3):904-13.

20. Evans JD, Gomez DR, Amini A, Rebueno N, Allen PK, Martel MK, et al. Aortic dose constraints when reirradiating thoracic tumors. Radiother Oncol. 2013;106(3):327-32. doi:10.1016/j.radonc.2013.02.002.

21. Nishimura S, Takeda A, Sanuki N, Ishikura S, Oku Y, Aoki Y, et al. Toxicities of organs at risk in the mediastinal and hilar regions following stereotactic body radiotherapy for centrally located lung tumors. J Thorac Oncol. 2014;9(9):1370-6. doi:10.1097/JTO.0000000000000260.

\section{Submit your next manuscript to BioMed Central and we will help you at every step:}

- We accept pre-submission inquiries

- Our selector tool helps you to find the most relevant journal

- We provide round the clock customer support

- Convenient online submission

- Thorough peer review

- Inclusion in PubMed and all major indexing services

- Maximum visibility for your research

Submit your manuscript at www.biomedcentral.com/submit
) Biomed Central 